\title{
Polar Run-Length Features in Segmentation of Retinal Blood Vessels
}

\author{
S. H. Rezatofighi,A. Roodaki \\ Control and Intelligent Processing Center \\ of Excellence, University of Tehran, \\ Tehran, Iran \\ h.tofighi@ece.ut.ac.ir
}

\author{
A. Pourmorteza \\ Control and Intelligent Processing Center \\ of Excellence, Univ. of Tehran, Tehran, \\ Iran \& Department of Biomedical \\ Engineering, Johns Hopkins School of \\ Medicine, Baltimore, MD, USA
}

\author{
H. Soltanian-Zadeh \\ Control and Intelligent \\ Processing Center of Excellence, \\ Univ. of Tehran, Tehran, Iran \& \\ Henry Ford Hospital, Detroit, \\ MI, USA.hamids@rad.hfh.edu
}

\begin{abstract}
Manual segmentation of retinal blood vessels in optic fundus images is a tiresome task. Several methods have previously been proposed for the automatic segmentation of retinal blood vessels. In this paper we propose a classifierbased method. First the images are preprocessed so that the within class variability of the vessel and background classes are minimized. Next, the image is scanned with a window of a certain size. Polar run-length matrices are simply created by transforming the windows into polar coordinates and then constructing conventional run length matrices. Two features are then extracted for each gray level value in the polar run length matrix. The feature vectors are then classified using a multilayer perceptron artificial neural network. The performance of the proposed method is compared with that of the human observers and with those methods previously reported in literature.
\end{abstract}

Keywords-retinal vessel segmentation; DoOG filters; polar transformation; run-length matrix;artificial neural network

\section{INTRODUCTION}

Eye is a window to the retinal vasculature, where the influence of the factors affecting the human body vasculature can be observed in vivo non-invasively. Early symptoms of systematic diseases can be detected by the assessment of retinal images. Furthermore, inspection of optic fundus photographs [1], [4], and flourocein images [2] may help to diagnose and monitor the progress of general diseases such as diabetes, hypertension, arteriosclerosis, cardiovascular diseases, stroke and eye diseases like retinopathy of prematurity [1], [2], [3]. Thus, the measurement and analysis of retinal blood vessels is of diagnostic value for a wide range of pathological states. However, manual analysis of the complex retinal blood vessel trees in fundus images is laborious and as the number of images increases, it may even be impossible. Segmentation of the vessels from the background is an initial requirement for the automatic assessment of retinal images. In classifier-based methods, various types of features are extracted from the image and the feature vectors are then introduced to a classifier to assign the corresponding pixel to a certain class or segment of the image. For example, in [4], wavelet transform features of an image are extracted and a Gaussian mixture model classifier is used for classification. In [2] a method based on multi-scale feature extraction is introduced to automatically segment the retinal blood vessels from the background. The method uses first and second derivatives of the intensity image, in a multiple pass region growing algorithm. The method in [5] starts with simple kernel-based preprocessing operations followed by a contrast enhancement step. Then a hidden Markov model-based classification is used to assign the pixels to vessel or background classes.

In this paper we have proposed a new classifier-based method mainly based on the features used to classify the pixels. Features extracted from the run-length matrix of a window of image can characterize its texture information. Here we exploit this ability of the run-length matrix to detect retinal blood vessels. Since the blood vessels tend to lie in different directions, the traditional run-length matrices, which encode the texture information in a few directions, are not efficient. In an attempt to overcome this problem the window of image is first transformed from Cartesian coordinates into polar coordinates.

A brief introduction to polar run-length features is provided in section 2 . Section 3 explains the preprocessing step. Section 4 includes the segmentation algorithm and necessary post-processing step to improve the results. The experimental results of the proposed method are presented in section 5. Section 6 is dedicated to discussion and conclusion.

\section{THE POLAR RUN-LENGTH}

In segmentation of retinal blood vessels, we are interested in features that provide efficient discriminatory information. Run-length matrix of an image has the capability of encoding its textural properties in the form of gray level runs.

In the feature extraction step, the image is swept by a window of a certain size and run length matrices of each window are formed. Next, features are extracted from the matrices and are assigned to the pixel located in the center of the sweeping window.

Traditional run length matrices are defined only in four directions. However, most of the vessels in the retinal images seem to lie in various directions. When the center of the sweeping window coincides with a pixel on a vessel, the vessels seems to extend in radial direction with respect to the 
center. Pixels on the vessels will share this property whereas those on the background do not have this radial symmetry. This radial symmetry suggests that most of the run length information of the window lie in radial and tangential directions as opposed to the conventional run length where the information is supposed to exist in the four basic directions.

In our proposed method, each window of the image is transformed into polar coordinates with its origin coinciding with the center of the window. Then run length matrices of radial direction i.e. horizontal direction in the polar image, are constructed and features can be extracted from the polar run length matrices (see Fig.1).

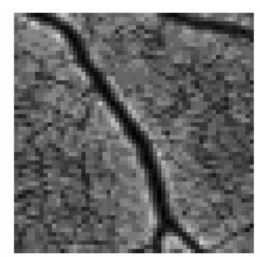

(a)

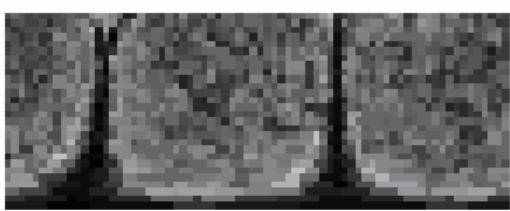

(b)
Figure 1. (a) A window of the preprocessed image, (b) its transformation in polar coordinates.

The polar transformed window could also be interpreted as a form of shape signature. A shape signature can be obtained by computing the distance of every point on the edge of the shape from a reference point. The downside of this description is that it only uses the data on the edge of the shape and no information is extracted from the inside of the shape. This exacts an edge detection step which can be timeconsuming and erroneous in the presence many grayscales and noise. Furthermore, the result does not contain any information about the internal features of the shape. The signature could be thought of as a transformation of the edge data from the Cartesian coordinates to a polar coordinate where the origin of the polar system coincides with the reference point of the signature. Instead of transforming the edge data, we have formed a complete transformation of the shape into the polar coordinates. The advantages are twofold: first, the whole data from the shape is taken into consideration; second, the resulted image is almost rotation invariant.

In our preliminary studies, the conventional run length features did not provide enough discriminatory power. Therefore we proposed two features for every gray level of the polar run length matrix. The first feature is the maximum number of occurrences multiplied by the length of the run with maximum occurrence. The second one is the sum of every occurrence multiplied by its corresponding run length. Thus for an image with 256 gray levels, 512 features are assigned to each pixel. These features, although chosen somewhat intuitively, are capable of discriminating between the vessel and background classes effectively.

\section{PREPROCESSING}

The preprocessing phase is aimed to reduce the unwanted effects of noise and background intensity variations, and to enhance the contrast between vessel and non-vessel pixels prior to feature extraction. Since the classifier will have to learn feature vectors from same classes in different, the images must share nearly similar brightness and contrast characteristics so that the within class variability of the feature vectors is minimized.

First step is to choose a monochromatic image that shows high contrast between vessel and non-vessel pixels. A Monte Carlo simulation of retinal vessel profiles [9] predicts that light observed from a retinal vessel at green band on an RGB color image is predominantly backscattered from the vessel or transmitted once through the vessel [2]. This is confirmed by experimental results in [1], [4], and [6]. Hence we have selected the green channel representation in our study. In an attempt to compensate for the contrast and brightness variations through the data set, the mean and standard deviation of the histograms of the images are changed so that they nearly match predefined values of mean $=0.5$ and $S=0.075$, where images have an intensity range of [0 1].

To compensate for the background intensity variations, an averaging kernel is convolved with the green channel. This convolution gives an approximation of the smooth background variations. Next, this approximation is subtracted from the green image. Size of the smoothing kernel is empirically selected to be $31 \times 31$.

To improve the discrimination between these thin vessels and the background noise, the normalized image is convolved with a set of line detection filters. Similar to kernels proposed in [1], first-order derivative filters, known as difference of offset Gaussians filters (DoOG filters), with prevailing responses to horizontal, vertical, and diagonal directions are used in this work. For each pixel, the maximum filter response is added to the normalized image. The DoOG filters have proved to be a good alternative to the more conventional solutions such as the Sobel kernels. Since they depend on larger kernels, these filters have greater immunity to noise.

Numerous methods for contrast enhancement of the retinal images are reported in the literature. A kernel-based local contrast enhancement technique is introduced in [3]. In [6] a number of contrast enhancement techniques, from the classic Histogram Equalization to newly developed Contourlet transform are discussed. Since the proposed algorithms are computationally intensive, a relatively simple, yet effective, method is chosen here. The significance of finer contrast enhancement techniques, however, is not denied.

Histogram of the background normalized image resembles a Gaussian distribution with a small variance where most of the pixels have midrange gray-levels. In an attempt to enhance the global contrast, the histogram is stretched by a sigmoid function whose inflection point is adaptively chosen to coincide with the peak of the histogram. Fig. 2 summarizes the results of the preprocessing step. 


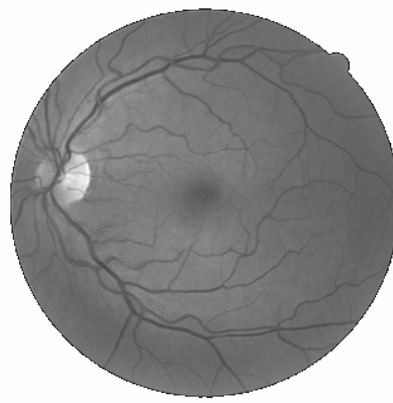

(a)

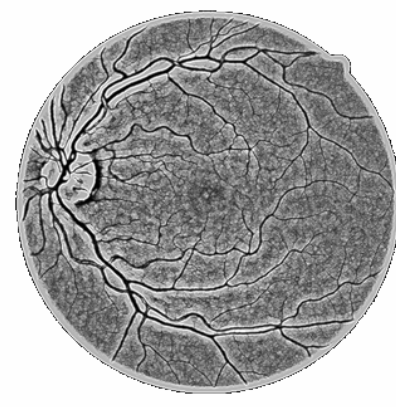

(b)
Fig. 2. (a) Green channel of image, (b) contrast enhanced image

\section{Segmentation AND Post-PROCESSING}

The green channel contents of the retinal image undergo the preprocessing step to compensate the smooth background variations, to enhance thin vessels, and to enhance the gray level contrast. The image is then scanned with a window and each window is transformed into polar coordinates; run length matrix corresponding to horizontal direction in the polar space is constructed and two features are extracted for every intensity value in the polar run length matrix. Thus for an image with 256 gray level intensity values, the feature vector assigned to every pixel will have 512 elements.

Henceforward the algorithm is a classification problem. Feature vectors from every pixel of the image need to be classified into one of the two classes of vessel or background. Here we use a multilayer perceptron (MLP) artificial neural network for the classification of feature vectors. The network is first trained with a fraction of retinal image data set using the class labels obtained from manual segmentation of retinal blood vessels. The trained network is then used for the classification of the rest of the images.

Presence of noise and contrast variations, along with fact that the trained classifier is not error-free, results in falsely detected blood vessels. Here we employ a post-processing step based on morphological operations to address this problem. First, the image is eroded using a structural element of a certain size. Next, connected components smaller than a specified size are removed. Finally the image is dilated using the same structural element that was used for eroding.

\section{EXPERIMENTAL RESULTS}

Our proposed method was tested on images of the publicly available DRIVE database [10]. The DRIVE database contains 40 color images of the retina, with $565 \times$ 584 pixels and 8 bits per color channel, in LZW compressed TIFF format. The images were originally captured from a Canon CR5 nonmydriatic 3 charge-coupled device (CCD) camera and were initially saved in JPEG-format. The database also includes binary images with the results of manual segmentation. These binary images have already been used as ground truth for performance evaluation of several vessel segmentation methods [10]. The 40 images were divided into a training set and a test set by the authors of the database. The results of the manual segmentation are available for all the images of the two sets. For the images of the test set, a second independent manual segmentation also exists.

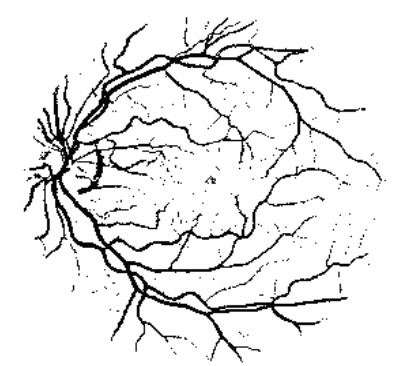

(a)

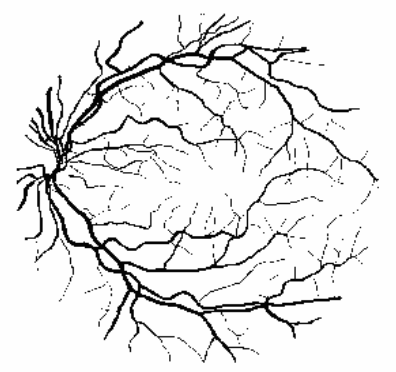

(b)
Fig. 3. Result of (a) Proposed algorithm, (b) Human observer

Segmentation accuracy is selected as performance measure to compare our results with previous retinal vessel segmentation algorithms. The accuracy is estimated by the ratio of the total number of correctly classified points (sum of true positives and true negatives) by the number of nonblack points in the image. The ground proof for computing the performance measures was the manual segmentation result. The values for the fraction of pixels erroneously classified as vessel pixels, false positive ratio (FPR), and the percentage of pixels correctly classified as vessel pixels, true positive ratio (TPR), are also reported.

We used the images in the test set of the DRIVE database to evaluate our algorithm. The images were initially preprocessed according to the algorithm discussed in section 3 . Then, the first image of the training set was used to train the network using the labels of the first set of manual segmentations of the DRIVE database. Next, the feature vectors from the normal images of the test set were classified by a three-layer MLP with 40, 20, and 1 neuron(s) in each layer. Last step included erosion, small connected component removal, and dilation to remove the falsely detected vessel pixels (see Fig. 3). Table 1 shows the results of our experiment along with those of other experiments in the literature carried out on the DRIVE database.

TABLE I. PERFORMANCE OF VESSEL SEGMENTATION METHODS

\begin{tabular}{cccc}
\hline Method & Accuracy & TPR & FPR \\
\hline $2^{\text {nd }}$ human observer & 0.9473 & 0.7761 & 0.0275 \\
Mendonça et al. [1] & 0.9452 & 0.7344 & 0.0236 \\
Jiang et al. [4] & 0.8911 & \multicolumn{2}{c}{ not reported } \\
Staal et al. [1] & 0.9442 & 0.7194 & 0.0227 \\
Niemeijer [1] & 0.9417 & 0.6898 & 0.0304 \\
Soares et al. [4] & 0.9466 & \multicolumn{2}{c}{ not reported } \\
Pourmorteza et al. [5] & 0.9401 & 0.7492 & 0.0384 \\
our proposed method & 0.9462 & 0.7358 & 0.0233 \\
\hline
\end{tabular}




\section{DISCUSSION AND CONCLUSION}

Our main purpose was to demonstrate the ability of polar run length features in providing discriminatory information for the segmentation of retinal blood vessels. The results are particularly comparable to previous classifier-based methods. In [4], all 20 images of the training set of the DRIVE database were used for training, with about one million feature vectors randomly selected; whereas in our work, the training data were extracted from one image. Thus the training time was reduced to almost one eighth of what was reported in [4]. However, the accuracies of these two methods are approximately equal. The simplicity of the feature vectors is also remarkable. Unlike [4] in which feature vectors are formed by Gabor transforms, our proposed feature vectors are merely made of sums and products of gray level runs. However, our proposed features are not as simple as those used in [5] in which sequences of intensities of pixels in a neighborhood were used as feature vectors. Hidden Markov models used in [5] were trained using Expectation Maximization (EM) algorithm. Authors stated that the EM training algorithm is very sensitive to the starting points and tends to fall into local minima. Then again, despite of the fact that MLP artificial neural networks have their pitfalls too, their implementation is straightforward and a number of effective training algorithms have been proposed to reduce the chance of falling in local minima.

Furthermore, the polar run length technique provides a new insight toward the use of run length methods. Its capability to extract features from radial and tangential directions makes it a valuable tool in characterization and segmentation of medical images which exhibit rotation invariance or radial symmetry e.g. intravascular ultrasound (IVUS), cytological, iris images, etc.

Like any other classifier based algorithm, the downside to our algorithm is that the information used for training the MLP is not accurate. In fact, the TPR and FPR for the human observers are 0.7761 and 0.0275 , respectively. Therefore, some training vectors erroneously labeled by human observers hinder appropriate training of the MLP classifier which leads to reduction in the TPR and increase in the FPR of the results.

\section{REFERENCES}

[1] M. Mendonça and A. Campilho, "Segmentation of Retinal Blood Vessels by Combining the Detection of Centerlines and Morphological Reconstruction”, IEEE Trans. Med. Imag., vol. 147, pp. 1200 -1213, 2006.

[2] M.E. Martinez-Prez, A.D. Hughes, S.A. Thom, A.A. Bharath, and K.H. Parker, "Segmentation of Blood Vessels from Red-Free and Fluoroscein Retinal Images”, Med. Image. Anal., vol. 11, pp. 47-61, 2007.

[3] K.A. Vermeer, F.M. Vos, H.G. Lemij, and A.M. Vossepoel, “A Model Based Method for Retinal Blood Vessel Detection”, Comput. Biol. Med, vol. 34, pp. 209-219, 2004.

[4] J.V.B. Soares, J.J.G. Leandro, R.M. Cesar Jr., H.F. Jelinek, and M.J. Cree, "Retinal Vessel Segmentation Using the 2-D Gabor Wavelet and Supervised Classification”, IEEE Trans. Med. Imag, vol. 25 , pp. 1214-1222, 2006

[5] A. Pourmorteza, S.H. RezaTofighi, A. Roodaki, A. Yazdani, and H. Soltanian-Zadeh, "Context-Dependent Segmentation of Retinal Blood Vessels Using Hidden Markov Models”, CSICC 2008, CCIS (in press). Springer, Heidelberg (2008).

[6] P. Feng, Y. Pan, B. Wei, W. Jin, and D. Mi, "Enhancing Retinal Images by the Contourlet Transform”, Pattern Recogn. Lett., vol. 28, pp. 516-522, 2007.

[7] A. Hoover, V. Kouznetsova, and M. Goldbaum, "Locating Blood Vessels in Retinal Images by Piecewise Threshold Probing of a Matched Filter Response”, IEEE Trans. Med. Imag., vol. 19, pp. 203219, 2000.

[8] S. Chaudhuri, S. Chatterjee, N. Katz, M. Nelson, and M. Goldbaum, "Detection of Blood Vessels in Retinal Images Using TwoDimensional Matched Filters”, IEEE Trans. Med. Imag., vol. 8, pp. 263-269, 1989.

[9] M. Hammer, S. Leistritz, L. Leistritz, and D. Schweitzer, "Light Paths in Retinal Vessel Oximetry”, IEEE Trans. Biomed. Eng., vol. 48, pp. 592-598, 2001.

[10] M. Niemeijer, B. vanGinneken,2002[Online]. Available: http://www.isi.uu.nl/Reseach/Databases/DRIVE/results.php 